Indian J Anim Health (2021), 60(2): 284-286

DOI: https://doi.org/10.36062/ijah.2021.03021

\title{
Temporo-mandibular joint luxation and its management in a cat
}

\author{
S. Ramankutty ${ }^{1 *}$, S. S. Nair ${ }^{1}$, S. Anoop ${ }^{1}$, A. Hansoge ${ }^{1}$, D. Jennes ${ }^{1}$ and K. D. J. Martin ${ }^{1}$ \\ ${ }^{1}$ Department of Veterinary Surgery and Radiology, College of Veterinary and Animal Sciences, \\ Veterinary and Animal Sciences University, Mannuthy, Thrissur- 680 651, Kerala, India
}

\begin{abstract}
Temporo-mandibular joint (TMJ) luxation in cats occurs secondary to trauma and is characterized by the inability to close the mouth due to mal-aligned dental arches. The condition, if not delayed or not associated with fractures, can be corrected even with a pen if a proper diagnosis has been made. A one year old female Persian cat was presented to the University Veterinary Hospital Mannuthy, Kerala Veterinary and Animal Sciences University, with a history of inability to close the mouth since last evening. TMJ luxation on the left side was diagnosed by physical examination and radiography. A plastic pen was kept as a fulcrum between the carnassial teeth of the affected side and realignment of the luxated joint was adopted and the animal had an uneventful recovery.
\end{abstract}

Key words: Cats, Fulcrum, Plastic pen, Temporo-mandibular luxation

A temporo-mandibular joint (TMJ) luxation is a condition in which the condylar process is completely dislocated from the mandibular fossa. It is usually traumatic in origin and can be associated with injury to other maxillofacial structures (Thatcher, 2017) and occur spontaneously as in open mouth jaw locking during yawning or vocalisation (Hsuan et al., 2017). Inability to close the mouth is a typical feature of temporo-mandibular joint luxation (Çetinkaya, 2012). The usual finding is a rostro dorsal luxation with the mandible shifted to the side opposite to the side of joint dislocation and it occurs when the mandible is completely dislocated from the mandibular fossa and is entrapped above the articular eminence (Thatcher, 2017). Caudoventral luxation can also occur rarely in case of fracture of retroarticular process. Fibrotic changes may occur in the temporo-mandibular joint as there is delay in correction and may prevent complete reduction even in cases managed acutely (Klima, 2007). Delay in managing the TMJ luxations can result in difficulty in correcting ankylosis (Meomartino et al., 1999). The method of correction described here is suitable for acute cranio-dorsal luxation of mandible not associated with any other skeletal abnormalities or fracture.
A one year old female Persian cat was presented to the University Veterinary Hospital Mannuthy, Kerala Veterinary and Animal Sciences University with the complaint that the animal was unable to close its mouth since last evening and the owner was unaware of the cause. On physical examination, the jaws were widely separated with the inability to close the jaws even with force (Fig. 1). The dorso-ventral radiograph of skull revealed luxation of left temporo-mandibular joint and displacement of the mandible. The condition was diagnosed asrostro-dorsal luxation of the left temporomandibular joint (Fig. 2).

The cat was anesthetized with a combination of butorphanol@0.2 mg/kg body weight (BW), midazolam@0.2 mg/kg BW, ketamine@10 mg/kg BW and dexmedetomidine@10 $\mu \mathrm{g} / \mathrm{kg}$ BW as a single injection intramuscularly. The onset of anaesthesia was achieved within 5 minutes and it lasted for 25 minutes. The animal was positioned in sternal recumbency. Complete oral examination was carried out to rule out additional trauma to teeth or other maxillofacial structures. A fulcrum was created by inserting a rough-surfaced plastic pen between the maxillary fourth premolar and the mandibular first molar, with gentle closing of mouth with manual pressure. The pen was then

*Corresponding Author, E mail: soumya@kvasu.ac.in 
Indian Journal of Animal Health, December, 2021

Temporo-mandibular luxation in cat

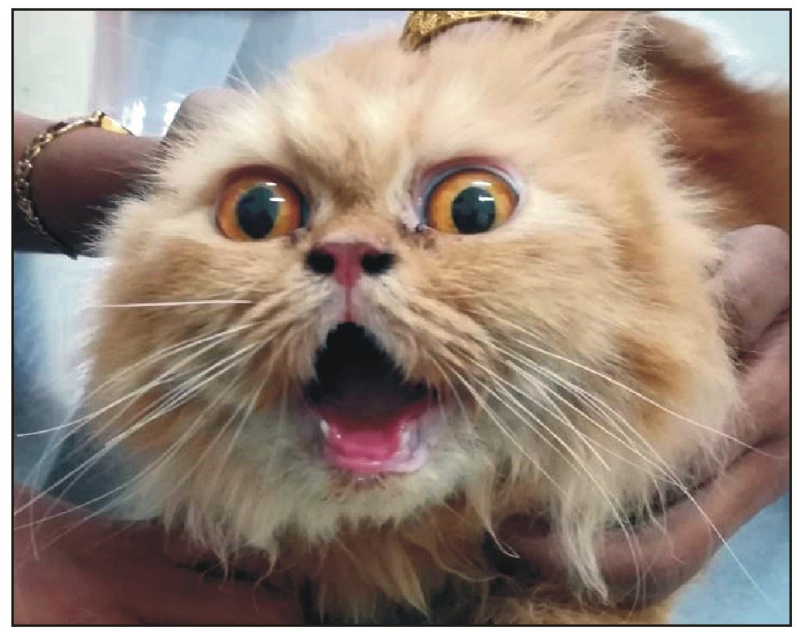

Fig. 1. Widely separated jaws with inability to close the mouth

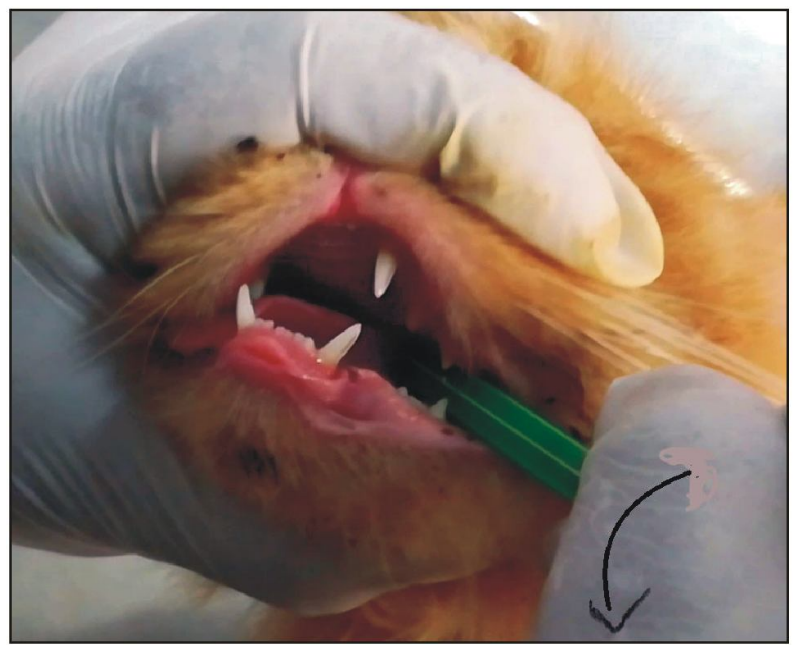

Fig. 3. Correction procedure by anticlockwise rotation of the pen

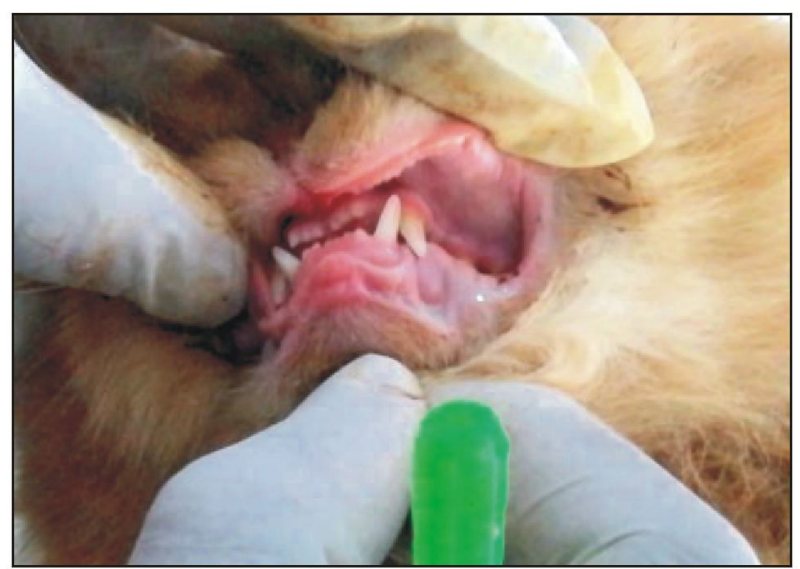

Fig. 5. Restoration of normal bite

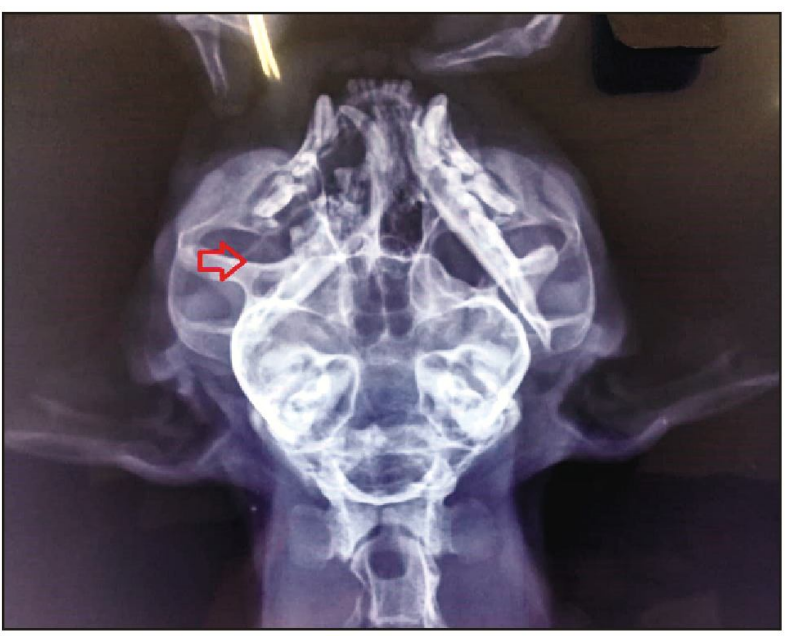

Fig. 2. Skull dorso-ventral radiograph showing deviation of mandible to the right

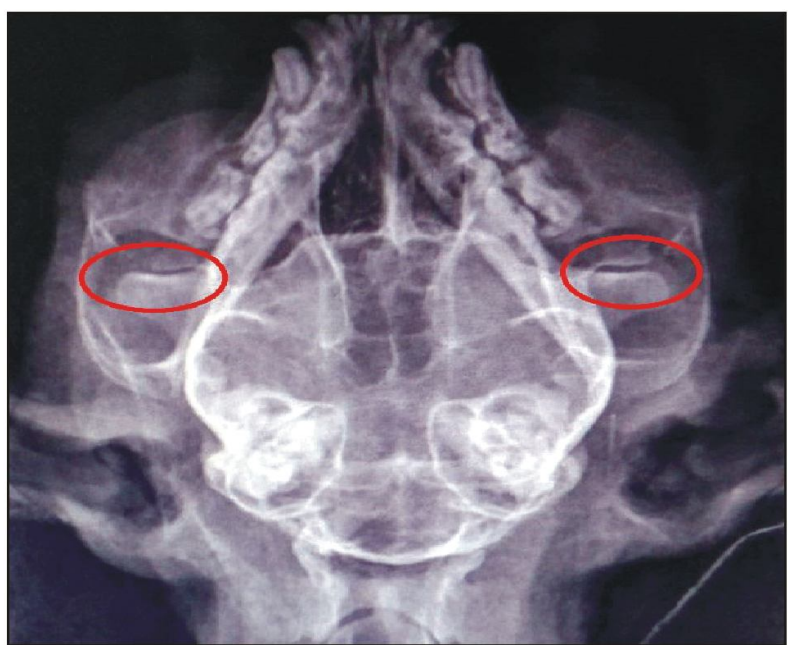

Fig. 4. Radiographic evidence of correction of the dislocation by the symmetry of structures

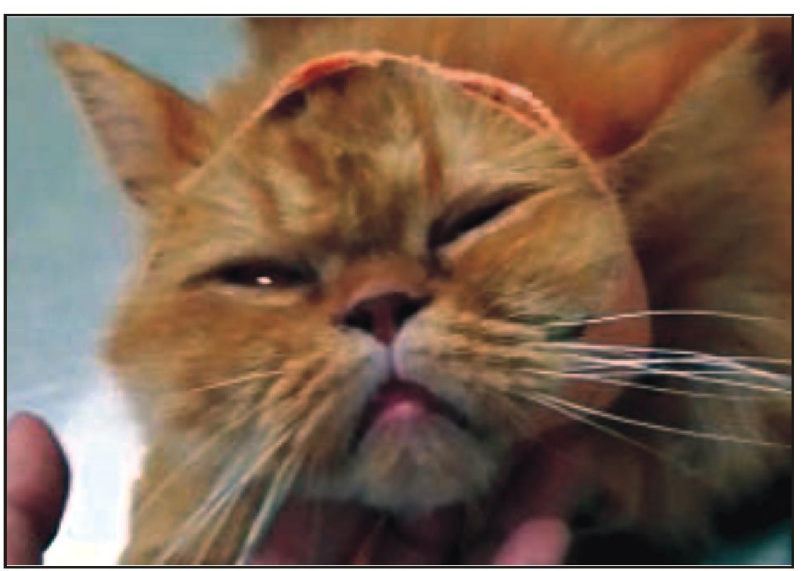

Fig. 6. Adhesive crepe bandage applied around the head 
rolled to facilitate reposition of the mandibular condyle caudally and ventrally into the mandibular fossa. As it was a rostro-dorsal luxation, anti-clockwise rotation of the pen was performed initially and the condylar process was positioned back over the mandibular fossa through a clockwise rotation of the pen (Fig. 3 ). The procedure resulted in reduction of the joint, normal alignment of the lower jaw, free movement of mandible and closure of the mouth. Post procedural radiograph confirmed the successful reduction of temporo-mandibular joint (Fig. 4). Restoration of normal bite was evident after the correction (Fig. 5). As it was a brachycephalic breed, an adhesive crepe bandage was applied around the head at nape to minimize the mandibular movements so that the animal could move its tongue to take liquid food (Fig. 6). The animal was treated with a course of meloxicam @ $0.2 \mathrm{mg} / \mathrm{kg}$ BW for next three days and recommended a soft diet for three weeks. The adhesive crepe bandage was removed after 10 days and the animal showed a complete recovery.

Temporo-mandibular joint (TMJ) luxations are common in cats and the usual type is rostral luxation resulting as frequent sequelae of maxillofacial injuries which result in displacement of condylar process dorsally and medially and is locked by the articular eminence (Çetinkaya, 2012; Thatcher, 2017). Caudoventral luxation can also occur rarely in case of fracture of retro-articular process which has a bad prognosis. TMJ luxation results in malalignment of dental arches and the presenting signs are characteristic malocclusion and the inability of the patient to completely close the mouth. When luxation is unilateral and rostrodorsal, the common radiographic feature is shifting of mandibles in opposite direction of luxation. Radiography in dorso-ventral or ventrodorsal view of skull and computed tomography proves to be diagnostic in this condition (Thatcher, 2017). The correction procedure done in this case was as described by Schulz (2007) and Thatcher (2017), in which a wooden pencil is used as fulcrum between mandibular firstmolar and maxillary premolar. Rotation of the pencil brought about repositioning of luxated condylar process into the mandibular fossa and was found to be highly effective for acute luxation of the TMJ. In the present case, a roughsurfaced plastic pen was used which provided adequate grip to do the procedure. Open reduction or condylectomy should be considered for recurrent cases of TMJ luxations (Klima, 2007). After reduction, application of a tape muzzle or inter arcade bonding using composite material can be done to prevent further movement of the joint if re-luxation is suspected (Legendre, 2005). In the present case, the adhesive crepe bandage was successful in providing adequate immobilization and the animal had an uneventful recovery. If closed reduction is unsuccessful or in cases of late presentation, open reduction or condylectomy is recommended (Thatcher, 2017). If a history of trauma is absent, open mouth jaw locking (OMJL) which happens during yawning or vocalisation can be suspected where in the coronoid process of the mandible gets displaced and locked lateral to zygomatic arch which prevents closing of mouth, and the correction occurs spontaneously or with minimum manipulation. Temporomandibular joint dysplasia can be a reason for the occurrence of TMJL and OMJL (Hsuan et al., 2017). The present case was that of a brachycephalic cat and brachycephalic cats have a shallow mandibular fossa and shorter mandibular head than mesocephalic group and thus they are predisposed for temporo-mandibular joint luxations according to Delesalle et al. (2020). According to Knight and Meeson, 2018, CT is more sensitive than plain radiography to detect fractures of skull in dogs and cats due to the complex anatomy of the skull and the lack of superimposition of structures and superior resolution achieved with CT. But in the present case, on physical examination, abnormal mobility or crepitus were not detected and the radiograph taken after the procedure did not show any fracture.

Conflict of interest: Authors have no conflict of interest in this study. 


\section{REFERENCES}

Çetinkaya MA, 2012. Temporomandibular joint injuries and ankylosis in the cat. Vet Comp Orthop Traumatol, 25(5): 366-374, doi: 10.3415/ VCOT-11-10-0146

Delesalle Q, Lopes AM, Gawor JP, Zani D, Pereira HM et al., 2020. Comparative morphometry of the temporomandibular joint in brachycephalic and mesocephalic cats using multislice CT and cone beam CT. J Feline Med Surg, 23(6): 507-512, doi: 10.1177/1098612X20960657

Hsuan L, Biller DS and Tucker-Mohl K, 2017. Open mouth jaw locking in a cat and a literature review. Israel J Vet Med, 72(4): 54-59

Klima LJ, 2007. Temporomandibular joint luxation in the cat. J Vet Dent, 24(3): 198-201, doi: 10.1177/ 089875640702400312

Knight R and Meeson RL, 2018. Feline head trauma: A CT analysis of skull fractures and their management in 75 cats. J Feline Med Surg, 21(12): 1120-1126, doi: 10.1177/1098612X 18819183

Legendre L, 2005. Maxillofacial fracture repairs. Vet Clin North Am Small Anim Pract, 35(4): 9851008, doi: 10.1016/j.cvsm.2005.03.003

Meomartino L, Fatone G, Brunetti A, Lamagna F and Potena A, 1999. Temporomandibular ankylosis in the cat: A review of seven cases. J Small Anim Pract, 40(1): 7-10, doi: 10.1111/j.17485827.1999.tb03245.x

Schulz K, 2007. Diseases of the joints. In: Small Animal Surgery ( $3^{\text {rd }}$ edn.) edited by: FossumTW, Mosby, Elsevier, St. Louis, Missouri, pp 11451315

Thatcher G, 2017. Temporomandibular joint luxation in the cat: diagnosis and management. Can Vet J, 58(9): 989-993

Received - 01.04.2021, Accepted - 25.06.2021, Published - 30.08.2021 (Online), 01.12.2021 (Print)

Section Editor: Prof. S. K. Nandi, Associate Editor 